\title{
Maximal Ratio Combining Using Channel Estimation in Chaos Based Pilot-Added DS-CDMA System with Antenna Diversity
}

\author{
Meher Krishna Patel, Stevan M. Berber, and Kevin W. Sowerby \\ Department of Electrical and Computer Engineering, The University of Auckland, Auckland, New Zealand \\ Correspondence should be addressed to Meher Krishna Patel; mpat260@aucklanduni.ac.nz
}

Received 1 July 2016; Accepted 8 August 2016; Published 9 January 2017

Academic Editor: Rafael Pérez-Jiménez

Copyright ( 2017 Meher Krishna Patel et al. This is an open access article distributed under the Creative Commons Attribution License, which permits unrestricted use, distribution, and reproduction in any medium, provided the original work is properly cited.

\begin{abstract}
This paper presents an adaptive multiuser transceiver scheme for DS-CDMA systems in which pilot symbols are added to users' data to estimate complex channel fading coefficients. The performance of receiver antenna diversity with maximal ratio combining (MRC) technique is analyzed for imperfect channel estimation in flat fading environments. The complex fading coefficients are estimated using least mean square (LMS) algorithm and these coefficients are utilized by the maximal ratio combiner for generating the decision variable. Probability of error in closed form is derived. Further, the effect of pilot signal power on bit error rate (BER) and BER performance of multiplexed pilot and data signal transmission scenario are investigated. We have compared the performance of added and multiplexed pilot-data systems and concluded the advantages of both systems. The proposed CDMA technique uses the chaotic sequence as spreading sequence. Assuming proper synchronization, the computer simulation results demonstrate the better bit error rate performance in the presence of channel estimator in the chaotic based CDMA system and the receiver antenna diversity technique further improves the performance of the proposed system. Also, no channel estimator is required if there is no phase distortion to the transmitted signal.
\end{abstract}

\section{Introduction}

The fundamental phenomenon which makes reliable wireless transmission difficult is time varying multipath fading. It is this phenomenon which makes wireless transmission a challenge when compared to fiber, coaxial cable, line-ofsight microwave, or even satellite transmission. Severe rapid fluctuation in received signal due to multipath environment makes it extremely difficult for the receiver to determine the transmitted signal [1]. In multipath fading environment, BER improvement of order $10^{-2}$ to $10^{-3}$ using typical modulation and coding schemes may require SNR improvement up to $10 \mathrm{~dB}$. Since there is power transmission limitation in wireless communication systems, hence BER improvement cannot be achieved by increasing the transmission power [2]. Spread spectrum techniques, channel estimation algorithms, and diversity techniques [3] are widely used methods for mitigating the effect of fading without increasing the transmission power.

CDMA system has received much attention in wireless communication system because the available spectrum is shared by all the users. In CDMA system each user is distinguished from others by uniquely defined spread sequence. Chaotic and Walsh sequences are widely used for identifying the correct user. Since interuser interference degrades the performance of CDMA system, the multiuser channel estimation gains a significant interest in recent years. Several methods have been proposed for estimating the channel based on short and long spreading codes [4-10].

In pilot assisted channel estimation [11-14] technique a predefined data sequence, known as pilot, is transmitted along with the actual message signal. These pilot sequences are used for estimating the fading coefficients and synchronization. Although there are various ways for transmitting the pilot signal, for example, sending pilot signal at the beginning or middle of each packet, in our work we have assumed that the pilot signal and actual users messages are transmitted simultaneously using separate spreading signals, which is a standard method in CDMA-2000 system. In this research work, it is assumed that the pilot and users data have the same power and they are not multiplexed but added to each other, 
which removes the assumption that pilot and users data have the same fading and noise effects. Additionally, a separate mathematical analysis has been done for different pilot and data powers and multiplexed pilot-data case.

Pilot signal and user's message have to be separated for estimating the channel coefficients and user's data; therefore spreading sequences should have impulse-like autocorrelation function and zero cross correlation values. Chaotic sequences possess good properties for pilot aided CDMA system. They are aperiodic sequences with mixed deterministicstochastic nature and are very sensitive to initial conditions. Therefore, infinite number of chaotic signals can be generated just from one chaotic map with impulse-like autocorrelation function with low cross correlation values [15]. Theoretical analysis of the chaos based CDMA systems has been done in many research works [16-20]. In these works channel coefficients are assumed to be real and positive. Complex channel gains are considered in [21-23] and perfect estimation of fading channel coefficients is assumed. Performance of these systems can degrade if fading coefficients are complex and the correct channel coefficients are not known at the receiver. Various adaptive equalization algorithms and corresponding BER performance for CDMA system with binary spreading codes have been investigated in [24-28] but none of these were used in chaos based CDMA system. Further, complex fading coefficients are estimated using Bayesian estimator in $[14,29]$ and with least mean square (LMS) in $[30,31]$ for chaos based CDMA systems.

Further, in [30,31], the received pilot signal is modified before using it for estimating the channel coefficients using LMS estimator. This paper shows analytically that the inclusion of spreading sequences increases the value of minimum mean square error for the LMS estimator, which can be minimized by normalizing the received pilot signal. Also, only added pilot-data systems are studied in [30, 31], whereas this paper compares, mathematically as well as using simulation results, the added and multiplexed pilot-data system. Moreover, perfect estimator and no estimator cases are studied in this paper, which demonstrate the need of channel estimator in the systems which have complex fading coefficients. Lastly, the first-order Markov channel model includes the Doppler effect in the channel; and we have shown the effects of Doppler frequency on BER performance of the system using simulation results.

Diversity is principally used to combat fading. Multipleantenna diversity is the most widely used diversity technique to mitigate the fading effect [32]. The method of fading mitigation using antenna diversity in chaos based CDMA system is discussed in [33] with known channel coefficients. The basic idea is that if different copies of the same signal are available then there is a high probability that at least one of them is of a good quality. Choosing the best copy and rejecting all others are not the optimal solution. This brings us to the problem of choosing the best way to combine all of them [11, 34]. MRC [35] technique is the most effective technique for combining. Channel estimator plays vital role in MRC because all the received signals must have the same phase before combining. Therefore channel estimators play dual role here; firstly they remove the fading effect from the received signals which are used for generating decision variable at each branch. Secondly they ideally maintain the zero phase signal at every branch; hence all the input signals to maximal ratio combiner have the same phase, to generate final decision variable.

The objective of this paper is to show the effect of imperfect channel estimation on the performance of chaos based DS-CDMA system in flat fading environment which can be extended for binary spreading sequence also. We have derived generalized BER expressions in closed form for imperfect channel estimation with receiver antenna diversity using MRC to optimize the decision. BER expression shows that the probability of error is inversely proportional cosine of the estimated phase error. LMS algorithm [36] is used for estimating the channel coefficients with the help of chaotic signals in CDMA system with receiver antenna diversity. All the received signals from different antennas are combined together using MRC method to generate BER curves and the results are compared with perfect channel estimation case. We assume a block fading channel; that is, length of data signal is less than the coherence time of the channel. Under these assumptions the estimation algorithm works in two modes, that is, training mode and tracking mode. In training mode channel coefficients are estimated with the help of known pilot signals. Then in tracking mode actual message is detected with the help of estimated channel coefficients. Finally we have shown that the BER expression derived in [16] is the special case of BER expression derived in this paper. During this complete process proper synchronization is assumed. Robust synchronization techniques for chaos based DS-CDMA system are shown in $[37,38]$ and effect of Rayleigh fading coefficients on synchronization process is analyzed in [17].

This paper is organized as follows. Section 2 presents the baseband representation of proposed chaos based DSCDMA system with channel estimation in the presence of complex noise and fading. Section 3 deals with the generation of decision variables. The analytical performance of MRC technique is shown in Section 4. Simulation results are presented in Section 5. Section 6 reports some concluding remarks.

\section{System Model}

Figure 1 shows the baseband representation of the chaos based CDMA system. The baseband signal $s_{k}^{(g)}$ for $g$ th user at $k$ th chip instant is given by

$$
s_{k}^{(g)}=\gamma_{i}^{(g)} x_{k}^{(g)},
$$

where $\gamma_{i}^{(g)}$ is $g$ th user symbol at $i$ th time instant, $x_{k}^{(g)}$ is $k$ th chip of $g$ th user chaotic spreading sequence or chip within an information bit (i.e., $k=1, \ldots, 2 \beta$ ), and $2 \beta$ is the spreading factor.

Similarly baseband pilot signal is represented by

$$
s_{k}^{(p)}=\gamma_{i}^{(p)} x_{k}^{(p)},
$$




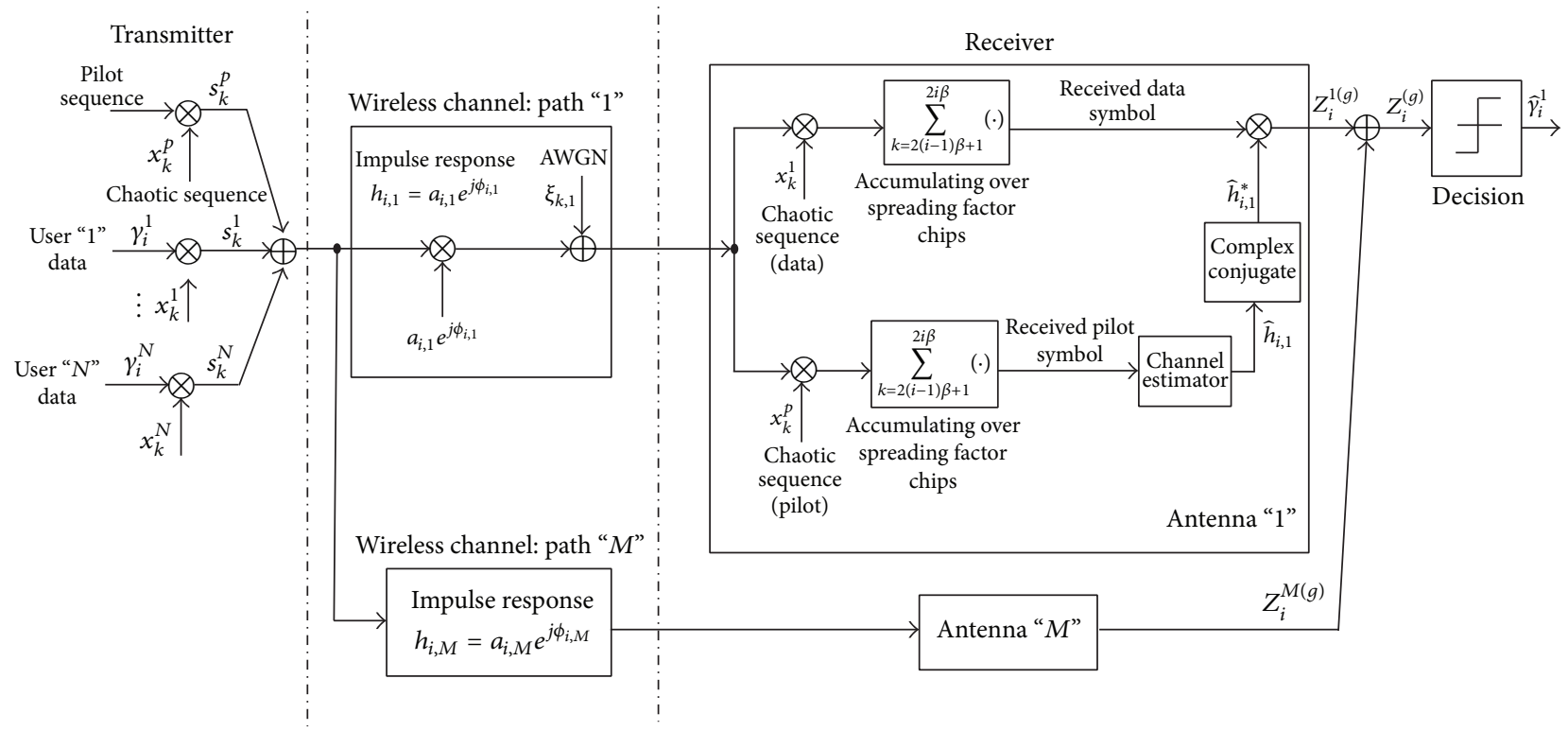

FIGURE 1: Block diagram of proposed system.

where $\gamma_{i}^{(p)}$ is the pilot symbol at ith time instant and $x_{k}^{(p)}$ is the chaotic spreading sequence for pilot. Pilot symbol is usually the series of ones; that is, $\gamma_{i}^{(p)}=1$. Here we assume that spreading sequence of pilot and data have the same chip power $P_{c}$. Bit error rate results for different pilot and data power for added pilot-data and multiplexed pilot-data case have been included in Appendix C.

The flat fading channel between transmitter and receiver antenna $m$ at time $i$ is denoted by complex variable $h_{i, m}$ which includes the amplitude variation $\left(a_{i, m}\right)$ and phase variations $\left(\phi_{i, m}\right)$ caused by the channel, expressed as

$$
h_{i, m}=a_{i, m} e^{j \phi_{i, m}}
$$

We assume that noise is affecting the signal on chip level, whereas fading is happening on bit level. Then $k$ th chip of received signal $r_{k}^{m(g)}$ at antenna $m$ of $g$ th user at time $i$ from $N$ transmission users is given by

$$
\begin{aligned}
r_{k}^{m(g)} & =h_{i, m} \sum_{j=p, j=1}^{N}\left(\gamma_{i}^{(j)} x_{k}^{(j)}\right)+\xi_{k, m} \\
& =a_{i, m} e^{j \phi_{i, m}} \sum_{j=p, j=1}^{N}\left(\gamma_{i}^{(j)} x_{k}^{(j)}\right)+\xi_{k, m},
\end{aligned}
$$

where $\xi_{k, m}$ denotes the complex additive white Gaussian noise for $m$ th receiving antenna with power spectral density equal to $N_{0}$. Clearly in the above equation every $2 \beta$ chip is representing one bit duration.

\section{Correlation Filter and Decision Variable}

In Figure 1, the received signal is processed at every antenna to separate the pilot and data symbols. Then the extracted pilot symbols are used to estimate the channel coefficients. These estimated channel coefficients are utilized by the maximal ratio combiner for generating the decision variable.

3.1. Pilot and Data Extraction. Correlation filter [39] is used to extract desired signal from the noisy signal. In Figure 1, received signal is multiplied by the locally generated pilot chaotic sequence and $g$ th user chaotic sequence to extract the pilot and $g$ th user data, respectively. After multiplication of chaotic sequences, the resultant sequences are accumulated over the $2 \beta$ interval. Hence the two correlator outputs are given by

$$
\begin{aligned}
z_{i, \text { data }}^{m(g)}= & \sum_{k=2(i-1) \beta+1}^{2 i \beta} a_{i, m} e^{j \phi_{i, m}}\left(\sum_{j=p, j=1}^{N}\left(\gamma_{i}^{(j)} x_{k}^{(j)}\right) x_{k}^{(g)}\right) \\
& +\sum_{k=1}^{2 \beta} \xi_{k, m} x_{k}^{(g)} \\
z_{i, \text { pilot }}^{m(g)}= & \sum_{k=2(i-1) \beta+1}^{2 i \beta} a_{i, m} e^{j \phi_{i, m}}\left(\sum_{j=p, j=1}^{N}\left(\gamma_{i}^{(j)} x_{k}^{(j)}\right) x_{k}^{(p)}\right) \\
& +\sum_{k=1}^{2 \beta} \xi_{k, m} x_{k}^{(p)},
\end{aligned}
$$

where $z_{i \text {,data }}^{m(g)}$ and $z_{i \text {,pilot }}^{m(g)}$ are the extracted data and pilot in $m$ th antenna branch at time $i$ of $g$ th user. 
3.2. Channel Estimation and Decision Variable. This extracted pilot is used as the input to the LMS filter and the resultant algorithm can be represented as follows:

$$
\begin{aligned}
e_{i} & =z_{i, \text { pilot }}^{m(g)}-\widehat{a}_{i, m} e^{j \widehat{\phi}_{i, m}} \sum_{k=2(i-1) \beta+1}^{k=2 i \beta}\left(x_{k}^{(p)}\right)^{2} \\
\widehat{a}_{i+1, m} e^{j \widehat{\phi}_{i+1}, m} & =\widehat{a}_{i, m} e^{j \widehat{\phi}_{i, m}}+\mu \sum_{k=2(i-1) \beta+1}^{k=2 i \beta}\left(x_{k}^{(p)}\right)^{2} e_{i},
\end{aligned}
$$

where $\widehat{a}_{i, m} e^{j \widehat{\phi}_{i, m}}$ are the estimated channel coefficients which are initialized with zero, $\mu$ is the step size, and $e_{i}$ is known as error function in the LMS filter. $z_{i \text {, pilot }}^{m(g)}$ and $\sum_{k=2(i-1) \beta+1}^{k=2 i \beta}\left(x_{k}^{(p)}\right)^{2}$ are known as desired signal and known signal in estimation theory [25]. The minimum mean square error (MMSE) $J_{\min }$ for this algorithm is (see Appendix A for derivation)

$$
J_{\min }=2 \beta a_{i, \mathrm{~m}}^{2} N P_{c}^{2}+2 \beta N_{0} P_{c}
$$

Channel coefficients can not be estimated properly with such an enormous amount of minimum mean square error. This value of $J_{\min }$ can be reduced by normalizing the desired signal and known signal by $\sum_{k=2(i-1) \beta+1}^{k=2 i \beta}\left(x_{k}^{(p)}\right)^{2}$. Thus new desired signal and known signal are $z_{i \text {,pilot }}^{m(g)} / \sum_{k=2(i-1) \beta+1}^{k=2 i \beta}\left(x_{k}^{(p)}\right)^{2}$ and 1 . In this case the new LMS algorithm is represented by

$$
\begin{aligned}
e_{i} & =\frac{z_{i, \text { pilot }}^{m(g)}}{\sum_{k=2(i-1) \beta+1}^{k=2 i \beta}\left(x_{k}^{(p)}\right)^{2}-\widehat{a}_{i, m} e^{j \widehat{\phi}_{i, m}}} \\
\widehat{a}_{i+1, m} e^{j \widehat{\phi}_{i+1, m}} & =\widehat{a}_{i, m} e^{j \hat{\phi}_{i}}+\mu e_{i} .
\end{aligned}
$$

The decision variable is obtained by multiplying the extracted data symbol $z_{i \text {,data }}^{m(g)}$ with the conjugate of the estimated channel coefficients. Hence the decision variable $Z_{i}^{m(g)}$ after processing the signal at antenna $m$ at time $i$ is given by

$$
Z_{i}^{m(g)}=z_{i, \text { data }}^{m(g)}\left(\widehat{a}_{i, m} e^{j \widehat{j}_{i, m}}\right)^{*}=z_{i, \text { data }}^{m(g)}\left(\widehat{a}_{i, m} e^{-j \widehat{\phi}_{i, m}}\right)
$$

where $*$ denotes the conjugation operation.

Putting value of $z_{i \text {,data }}^{m(g)}$ from (5) to the above equation and after separating mathematical equations of various terms, that is, desired signal, multiuser interference, and channel noise, we get the above equation in the following form:

$$
\begin{aligned}
& Z_{i}^{m(g)} \\
& =\underbrace{a_{i, m} \widehat{a}_{i, m} \mathrm{e}^{j\left(\phi_{i, m}-\widehat{\phi}_{i, m}\right)} \sum_{k=2(i-1) \beta+1}^{2 i \beta} \gamma_{i}^{(g)}\left(x_{k}^{(g)}\right)^{2}}_{\text {Desired Signal }} \\
& +\underbrace{a_{i, m} \widehat{a}_{i, m} e^{j\left(\phi_{i, m}-\widehat{\phi}_{i, m}\right)} \sum_{j=2(i-1) \beta+1}^{2 i \beta} \sum_{j=1, j \neq g}^{N}\left(\gamma_{i}^{(j)} x_{k}^{(j)}\right) x_{k}^{(g)}}_{\text {Multiuser Interference }} \\
& +\underbrace{\widehat{a}_{i, m} e^{-j i \beta} \widehat{\phi}_{i, m} \sum_{k=2(i-1) \beta+1} x_{k}^{(g)}}_{\text {Channel Noise }} .
\end{aligned}
$$

\section{Performance Analysis of MRC Technique}

In MRC technique all intermediate decision variables $Z_{i}^{m(g)}$, $m=1, \ldots, M$ are added together to generate the final decision variable, which is given by

$$
Z_{i}^{(g)}=\operatorname{Re}\left\{\sum_{m=1}^{M} Z_{i}^{m(g)}\right\}
$$

where $Z_{i}^{(g)}$ is the final decision variable used for symbol detection and $\mathrm{Re}$ is the real part of complex function.

Putting value of $Z_{i}^{m(g)}$ from (11) to the above equation, we get final decision variable as follows:

$$
\begin{aligned}
& Z_{i}^{(g)} \\
& =\operatorname{Re}\left\{\sum_{m=1}^{M} a_{i, m} \widehat{a}_{i, m} e^{j\left(\phi_{i, m}-\widehat{\phi}_{i, m}\right)} \sum_{k=2(i-1) \beta+1}^{2 i \beta} \gamma_{i}^{(g)}\left(x_{k}^{(g)}\right)^{2}\right. \\
& +\sum_{m=1}^{M} a_{i, m} \widehat{a}_{i, m} e^{j\left(\phi_{i, m}-\widehat{\phi}_{i, m}\right)} \\
& \times \sum_{k=2(i-1) \beta+1}^{2 i \beta} \sum_{\substack{j=1, j \neq g \\
j=p}}^{N}\left(\gamma_{i}^{(j)} x_{k}^{(j)}\right) x_{k}^{(g)} \\
& \left.+\sum_{m=1}^{M} \widehat{a}_{i, m} e^{-j \widehat{\phi}_{i, m}} \sum_{k=2(i-1) \beta+1}^{2 i \beta} \xi_{k, m} x_{k}^{(g)}\right\}
\end{aligned}
$$




$$
\begin{aligned}
= & \sum_{m=1}^{M} a_{i, m} \widehat{a}_{i, m} \cos \left(\phi_{i, m}-\widehat{\phi}_{i, m}\right) \sum_{k=2(i-1) \beta+1}^{2 i \beta} \gamma_{i}^{(g)}\left(x_{k}^{(g)}\right)^{2} \\
& +\sum_{m=1}^{M} a_{i, m} \widehat{a}_{i, m} \cos \left(\phi_{i, m}-\widehat{\phi}_{i, m}\right) \\
& \times \sum_{k=2(i-1) \beta+1}^{2 i \beta} \sum_{\substack{j=1, j \neq g \\
j=p}}^{N}\left(\gamma_{i}^{(j)} x_{k}^{(j)}\right) x_{k}^{(g)} \\
& +\sum_{m=1}^{M} \widehat{a}_{i, m} \cos \left(\widehat{\phi}_{i, m}\right) \sum_{k=2(i-1) \beta+1}^{2 i \beta} \operatorname{Re}\left(\xi_{k, m}\right) x_{k}^{(g)} \\
& +\sum_{m=1}^{M} \widehat{a}_{i, m} \sin \left(\widehat{\phi}_{i, m}\right) \sum_{k=2(i-1) \beta+1}^{2 i \beta} \operatorname{Im}\left(\xi_{k, m}\right) x_{k}^{(g)},
\end{aligned}
$$

where $\operatorname{Re}\left(\xi_{k, m}\right)$ and $\operatorname{Im}\left(\xi_{k, m}\right)$ are the real and imaginary parts of complex AWGN noise. Sign of this decision variable is the estimated symbol; that is,

$$
\widehat{\gamma}_{i}^{(g)}=\operatorname{sign}\left(Z_{i}^{(g)}\right) .
$$

The probability of error $\left(P^{(g)}\left(a_{i}\right)\right)$ for $i$ th bit of $g$ th user is given by [15]

$$
\begin{aligned}
P^{(g)}\left(a_{i}\right)= & \frac{1}{2} \operatorname{Pr}\left(Z_{i}^{(g)}<0 \mid \gamma_{i}^{(g)}=1\right) \\
& +\frac{1}{2} \operatorname{Pr}\left(Z_{i}^{(g)} \geq 0 \mid \gamma_{i}^{(g)}=-1\right) \\
= & \frac{1}{2} \operatorname{erfc}\left(\frac{E\left[Z_{i}^{(g)} \mid \gamma_{i}^{(g)}=1\right]}{\sqrt{2 \operatorname{var}\left[Z_{i}^{(g)} \mid \gamma_{i}^{(g)}=1\right]}}\right),
\end{aligned}
$$

where Pr and erfc are the probability operations and complementary error function, respectively.

Solving the above equation (see Appendix B for derivation) and omitting the subscript $i$ that is related to bit under investigation, we have the final equation of $P^{(g)}(a)$ as follows:

$$
\begin{gathered}
P^{(g)}(a)=\frac{1}{2} \operatorname{erfc}\left\{\left[\frac{\psi}{\beta}+\frac{N}{\beta}+\left(\left(\frac{E_{b}}{N_{0}}\right)\right.\right.\right. \\
\left.\left.\cdot \frac{\left\{\sum_{m=1}^{M} a_{m} \widehat{a}_{m} \cos \left(\phi_{m}-\widehat{\phi}_{m}\right)\right\}^{2}}{\sum_{m=1}^{M} \widehat{a}_{m}^{2}}\right)^{-1}\right\},
\end{gathered}
$$

where $\psi=\operatorname{var}\left[\left(x_{k}^{(g)}\right)^{2}\right] / P_{c}^{2}, P_{c}=E\left[\left(x_{k}^{(g)}\right)^{2}\right]$, and $E_{b}=2 \beta P_{c}$.

Hence the probability of error depends on the number of users $(N)$, spreading factor $(2 \beta)$, estimated channel coefficients $\left(\widehat{a}_{m}, \widehat{\phi}_{m}\right)$, the signal to noise ratio $\left(E_{b} / N_{0}\right)$ and total number of receiving antennas $(M)$. Clearly probability of error increases as the number of users in the system increases and decreases as the spreading factor, signal to noise ratio, and/or number of receiving antennas increases. Further, the error probability depends on the cosine value of the difference between estimated phase and actual phase of the complex channel coefficients. Therefore the performance of the system is greatly dependent on the performance of the channel estimation algorithms. From this expression it is clear that the probability of error is minimum for perfect channel estimation where the difference between actual and estimated phase is zero and then increases gradually as the difference increases.

For perfect channel estimation case (17) reduces to

$$
\begin{aligned}
P^{(g)}(a) & \\
& =\frac{1}{2} \operatorname{erfc}\left\{\left(\frac{\psi}{\beta}+\frac{N}{\beta}+\left(\sum_{m=1}^{M} a_{m}^{2} \frac{E_{\mathrm{b}}}{N_{0}}\right)^{-1}\right)^{-1 / 2}\right\} .
\end{aligned}
$$

If we assume that fading coefficients are real and positive (i.e., $\phi_{m}=0$ with no channel estimator in the system (i.e., $\left.\hat{h}_{i, m}=1 e^{0}\right)$, then we can neglect the pilot processing part in Figure 1 and total numbers of interuser interferers are reduced to $(N-1)$ in the absence of pilot signal. In this case (17) will become

$$
\begin{aligned}
P^{(g)}(a) & \\
& =\frac{1}{2} \operatorname{erfc}\left\{\left(\frac{\psi}{\beta}+\frac{(N-1)}{\beta}+\left(a_{1}^{2} \frac{E_{b}}{N_{0}}\right)^{-1}\right)^{-1 / 2}\right\} .
\end{aligned}
$$

Thus the above BER expression which is derived in [16] and used in research works $[16,17]$ is a special case of $(17)$. From (17) and (19) we can see that the performance of the system deteriorates significantly in the presence of complex channel coefficients. Removing pilot interference term from (18), that is, changing $N$ to $(N-1)$ and assuming only one receiving antenna, then from (18) and (19) we can observe that, for real fading coefficients, that is, when channel is not causing any phase distortion, the BER expression of the no estimator case is the same as the perfect estimator case. Therefore no channel estimator is required for real fading coefficients.

In general, the BER of the system $\left(P_{\mathrm{ff}+\mathrm{AWGN}}^{(\mathrm{g})}\right)$ can be expressed as [40]

$$
P_{\mathrm{ff}+\mathrm{AWGN}}^{(g)}=\int_{0}^{\infty} P^{(g)}(a) p(a) d a,
$$

where the subscript ff denotes the flat fading and $p(a)$ is the probability density function of Rayleigh distribution.

\section{Simulation Results}

Several cases are simulated to demonstrate the performance of the proposed pilot-added CDMA system with BPSK signaling. In the simulation we have used the first-order Markov process to model the fading process of channel [5, $41,42]$, which is described as

$$
h_{i+1, m}=\alpha_{m} h_{i, m}+v_{i, m}, \quad m=1, \ldots, M,
$$




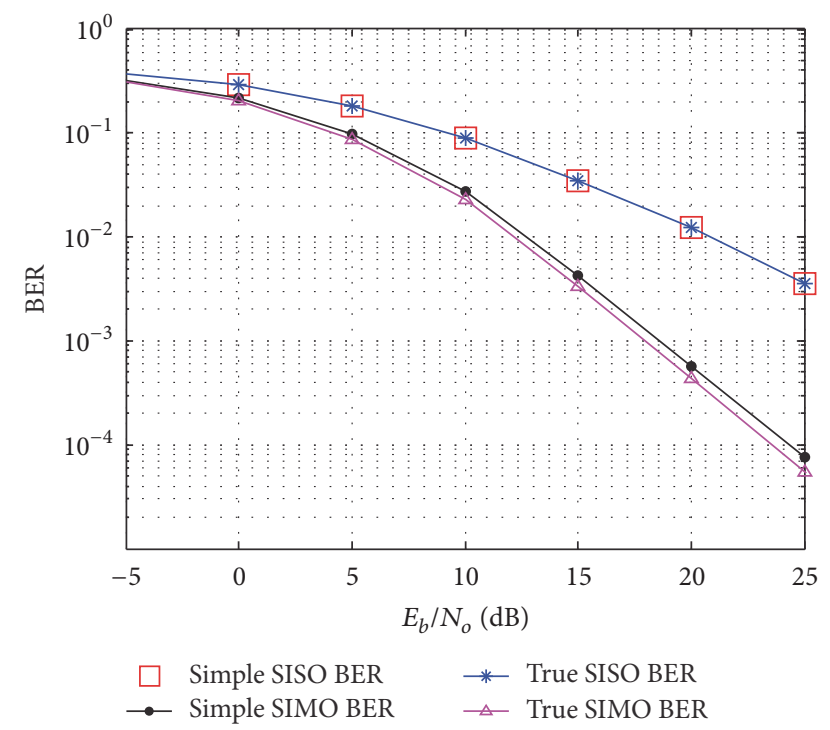

FIGURE 2: Simulated BER comparison of no channel estimator case and perfect estimator case for real fading coefficients in 1 user and pilot system with added pilot-data case, $f_{d} T_{s}=0.01$ and $2 \beta=50$.

where $v_{i, m}$ is the complex Gaussian process for antenna $m$ at time $i$. In the simulation it is assumed that fading processes are independent to each other with unit variances. $\alpha_{m}$ is the correlation coefficient that depends on maximum Doppler frequency $f_{d}$ and is defined as

$$
\alpha_{m}=J_{0}\left(2 \pi f_{d} T_{s}\right),
$$

where $J_{0}(\cdot)$ is the Bessel function of first kind and zerothorder and $T_{s}$ is the signaling rate.

In the simulation, the value of the spreading factor $2 \beta$ is 50. In LMS algorithm, the order of the filter is one and the step size $\mu$ is set to 0.9 . We have chosen the following Chebyshev polynomial function to generate the chaotic sequence because this chaotic generator outperforms many other chaotic sequence generators [43].

$$
x_{k}^{(j)}=1-2\left(x_{k-1}^{(j)}\right)^{2}, \quad j=(1, \ldots, N), p,
$$

where $x_{k}^{(j)}$ denotes the $k$ th chip value of $j$ th user and pilot.

In the simulation results SISO (single input, single output) and SIMO (single input, multiple output) represent the one receiving and two receiving antennas, respectively, with one transmitting antenna. We have used the MRC technique to generate the final BER curve in SIMO case throughout the simulation. Similarly "LMS BER," "True channel BER," and "Simple BER" represents the bit error rate with LMS channel estimation, perfect channel estimation, and no channel estimation case respectively.

Figure 2 compares the performance of no estimator case with perfect estimator case for real fading coefficients with one user and pilot signal and having fading rate of $f_{d} T_{s}=$ 0.01 . For simulation, the real fading coefficients are generated by taking absolute value of (21). For this case the performance of the system used in $[16,17]$ is matched with the perfect

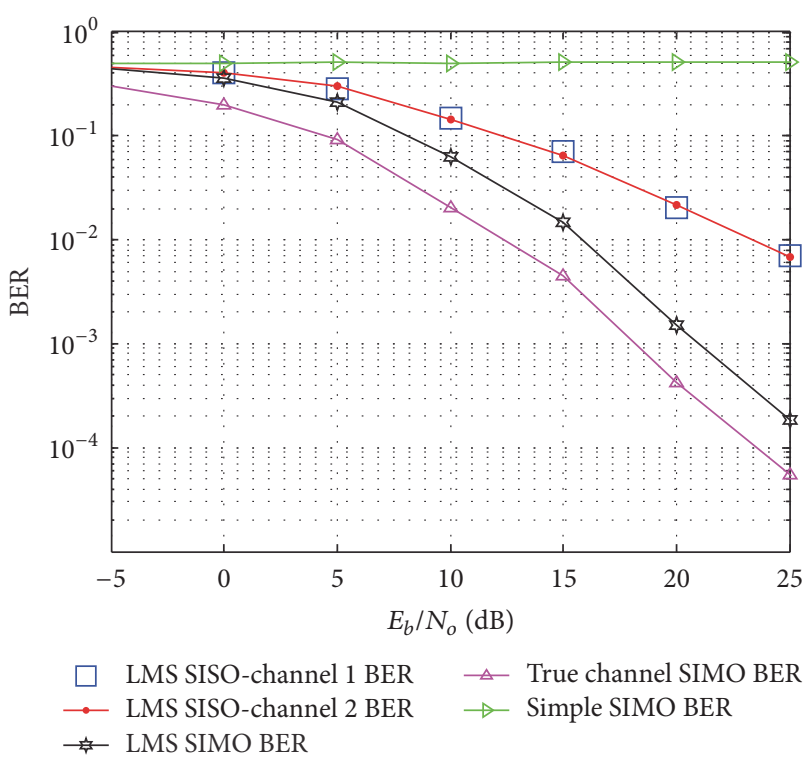

FIGURE 3: Simulated BER comparison of various methods in chaos based CDMA system for complex fading coefficients in 1 user and pilot system with added pilot-data case, $f_{d} T_{s}=0.01$ and $2 \beta=50$.

estimator case. Also there is a significant gain in the BER performance with two receiving antennas' diversity system over single antenna system.

Figure 3 demonstrates the BER comparison between various cases with one and two receiving antennas for $f_{d} T_{s}=$ 0.01 in the presence of one user and pilot signal with complex fading coefficients. In this case the BER for no estimator case is 0.5 for complete SNR range, which was quite good for real fading coefficients. This is happening because, for complex fading coefficients, no estimator case is not considering the phase change due to channel and simply giving a random guess about the transmitted bit, which has the probability of 0.5. From the plot it is clear that the channel estimator improves the performance of the proposed CDMA system. In this figure "LMS SISO-channel 1" and "LMS SISO-channel 2 " denote the BER due to individual antenna processing. Almost $5 \mathrm{~dB}$ performance improvement is achieved using $\mathrm{MRC}$ technique at $\mathrm{SNR}=15 \mathrm{~dB}$ with two receiving antennas as compared to one antenna system. This performance is further improved for higher SNR conditions.

Figure 4 shows the performance of two receiving antennas' diversity system for different fading rates for 1 user and pilot system. There is almost $2 \mathrm{~dB}$ performance decrement in BER performance with increase in fading rate from 0.01 to 0.1 .

Figure 5 depicts the performance of LMS estimator in two receiving antennas' diversity system for different number of users. The performance of the system degrades as the number of users increases.

Figure 6 illustrates that the performance of two receiving antennas' diversity system improves with higher values of spreading factor. On comparing this result with Figure 3, we can observe that the performance of system with 10 users with higher spreading factor, that is, 300 , is equivalent 


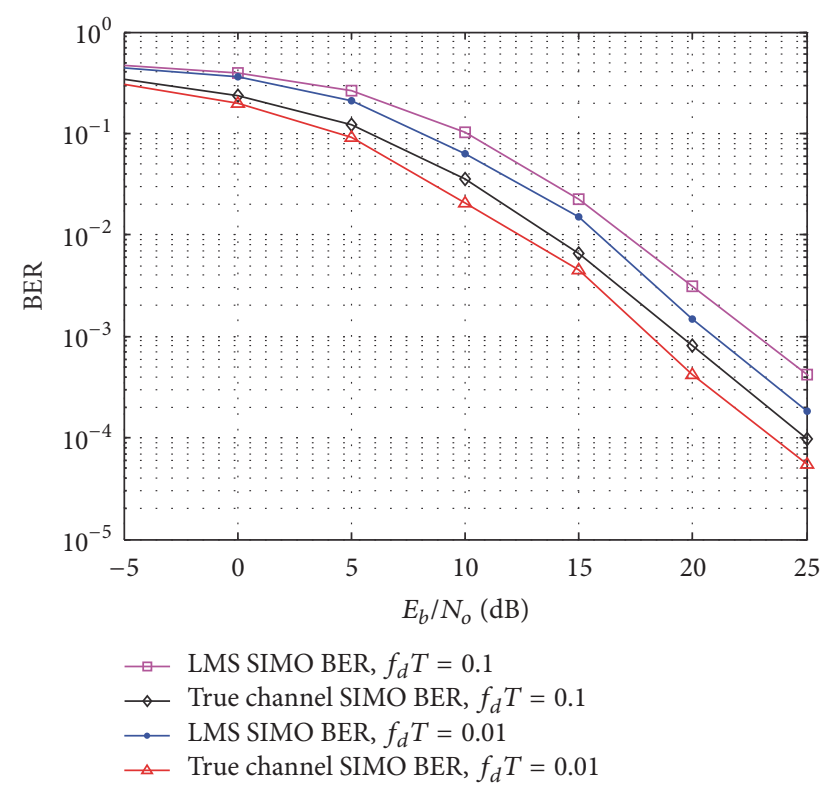

FIGURE 4: Simulated BER comparison of different fading rates with two receiving antennas' diversity in chaos based CDMA system for 1 user and pilot system with added pilot-data case, $2 \beta=50$.

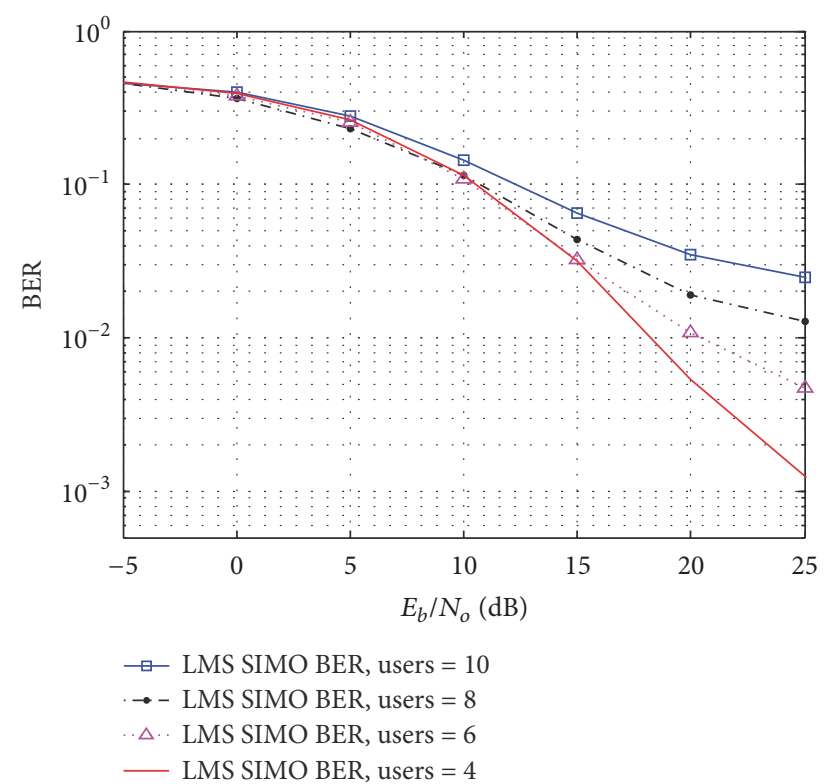

FIgURE 5: Simulated BER comparison of different number of users and pilot for two receiving antennas' diversity in chaos based CDMA system for added pilot-data case, $f_{d} T_{s}=0.1$ and $2 \beta=50$.

to performance of the system with monouser with lower spreading factor value, that is, 50 .

Effect of higher power of pilot is shown in Figure 7. Here we assumed that pilot power is $6 \mathrm{~dB}$ higher than the data and $f_{d} T_{s}=0.1$. It can be seen in the BER curves of estimation cases that there is an intersection point between equal pilotdata and higher pilot-data power case. Before intersection point, that is, at low SNR conditions, the performance of the higher pilot power case has better performance than equal

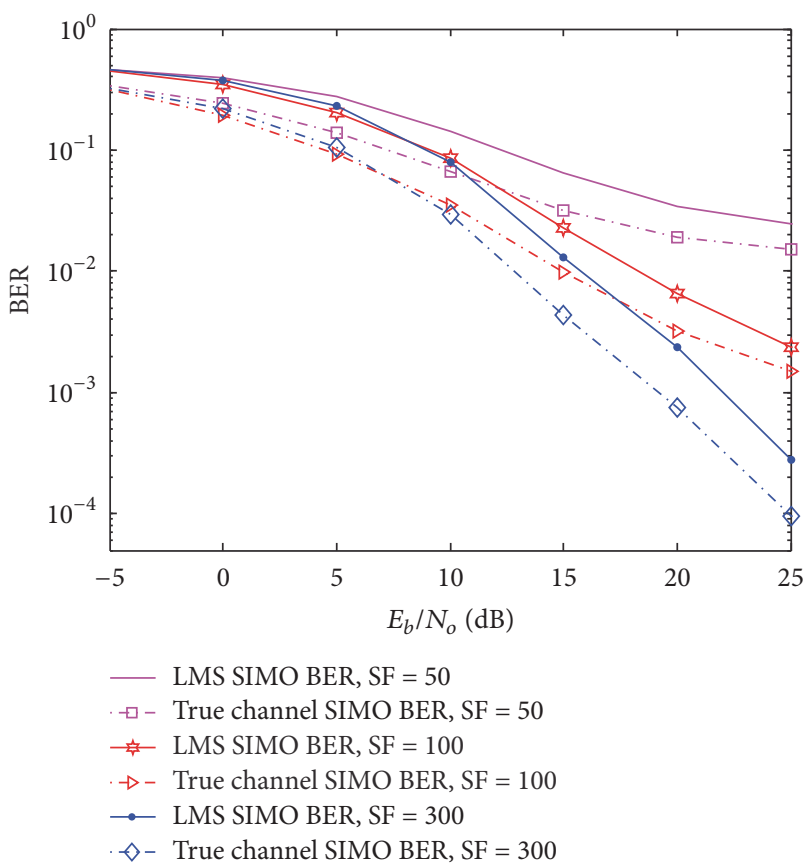

FIgURE 6: Simulated BER comparison of different values of spreading factor (SF) for 10 users and pilot for two receiving antennas' diversity in chaos based CDMA system for added pilot-data case, $f_{d} T_{s}=0.1$.

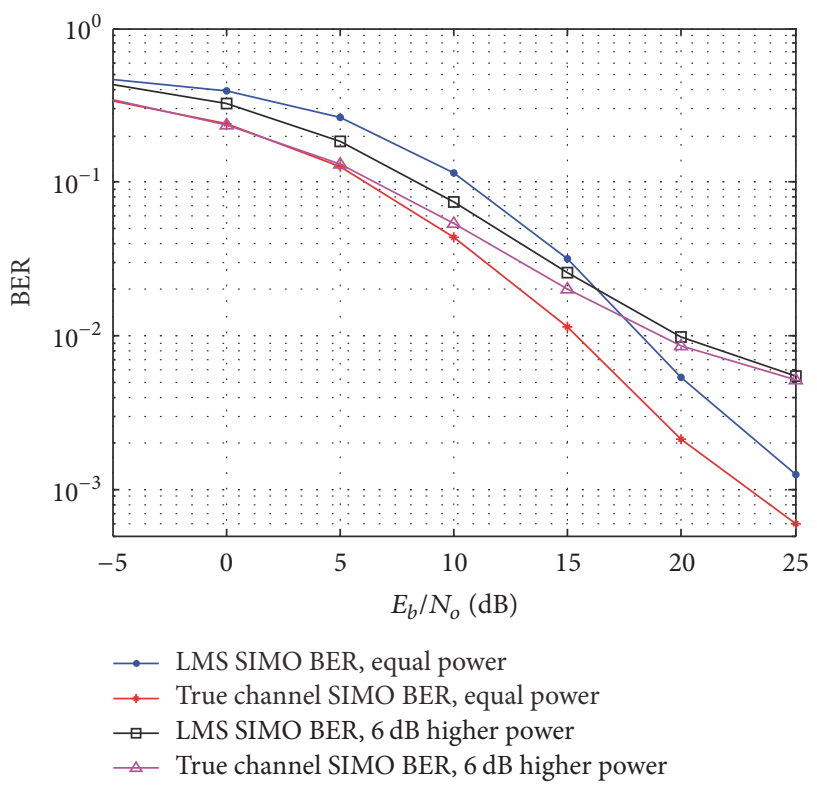

FIGURE 7: Simulated BER comparison of different pilot power with two receiving antennas' diversity in chaos based CDMA system for 4 users and pilot system with added pilot-data case, $f_{d} T_{s}=0.1$ and $2 \beta=50$.

power case and the performance degrades after the intersection points. From (C.1) it can be observed that pilot power increases the interuser interference term, but at the same time performance of the channel estimator improves because of higher pilot power. This performance improvement compensates the interuser interference caused by higher pilot power. 


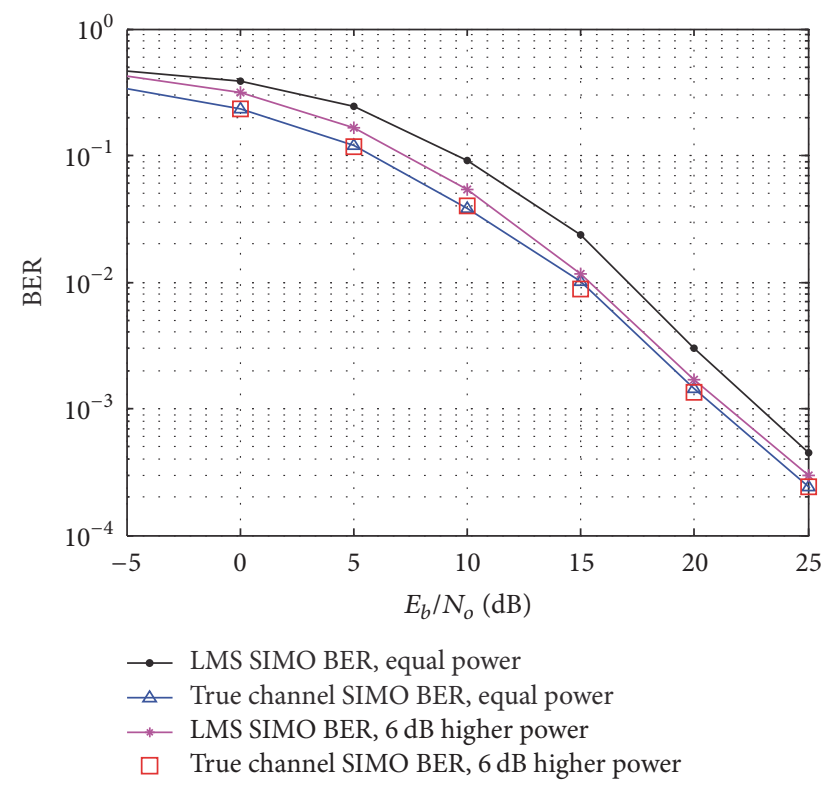

FIGURE 8: Simulated BER comparison of different pilot power with two receiving antennas' diversity in chaos based CDMA system for 4 users and pilot system with multiplexed pilot-data case, $f_{d} T_{s}=0.1$ and $2 \beta=50$.

But at higher SNR, the estimation algorithm can achieve minimum mean square value at low pilot power; therefore higher power is only producing the interuser interference. Since the higher pilot power transmission is not required at high SNR condition, hence this performance degradation can be improved by controlling the power transmission.

Finally in Figure 8, multiplexed pilot-data scenario for two receiving antennas is simulated with four users and pilot signal for $f_{d} T_{s}=0.1$. If we assume that pilot and data have the same fading and noise effect, then the performance of this system improves with increase in the pilot power transmission because the pilot is not creating any interuser interference. Similarly for perfect channel estimator case, this system has the same BER curves for higher power transmission and equal power transmission cases. Also, for higher pilot power transmission the BER performance of the system with LMS estimator is nearly equal to the perfect estimator case. But this performance improvement can degrade if the pilot and data do not have the same fading and noise effect.

\section{Conclusion}

In this paper, we propose the pilot-added transceiver for CDMA system in flat fading channel in which chaotic sequences are used for spreading the data symbols of various users. In simulation, performance of LMS channel estimation algorithm is compared with the perfect estimator case and with simple CDMA system in which no estimation algorithm is used, for the same pilot and data powers. There is huge BER performance improvement in the proposed CDMA system using channel estimation algorithm. However, for real fading coefficients, that is, when channel is not causing any phase distortion, then the BER expression of no estimator case is the same as the perfect estimator case as shown in simulation results. Therefore no channel estimator is required for real fading coefficients. The performance of the proposed system is then compared with the two receiver antennas' diversity system. Simulation results show that there is further improvement in the system using diversity technique. We have also verified that BER performance is slightly better for low Doppler spreading. It is shown that there is an increase in bit error rate with increase in pilot power, but this performance degradation is compensated by the performance improvement of the estimator at low SNR conditions. Since the high transmission pilot power is not required for high SNR conditions, therefore if the transmission power is controlled for high SNR conditions, then the proposed system's performance can be improved for high SNR case also. Separate mathematical equation is derived for the multiplexed pilot-data case. In this case we assume that pilot and data have the same fading effects. For this system the BER equation does not depend directly on the pilot power, but the performance of such system increases with increase in pilot power because the performance of estimator increases with power, which results in improvement in BER. Further the simulation results show that the BER performance of multiplexed data-pilot system using LMS channel estimator is closely matched to the perfect channel estimation case when pilot power is $6 \mathrm{~dB}$ higher than the data signal power. In final conclusion we can say that, for multiplexed pilot-data case, if pilot and data symbols do not have the same fading and noise effects then the added pilot-data system performs better than the multiplexed system and vice versa.

\section{Appendix}

\section{A. Derivation of LMS Error (See (8))}

Minimum mean square error of estimator is the error produced by the Wiener Filter when it is perfectly matched to the system model [36]. In this case, the minimum mean square error will be the variance of the noise and interuser interference. Rewriting (6) we have

$$
\begin{aligned}
z_{i, \text { pilot }}^{m(g)}= & a_{i, m} e^{j \phi_{i, m}} \gamma_{i}^{(p)} \sum_{k=2(i-1) \beta+1}^{2 i \beta}\left(x_{k}^{(p)}\right)^{2} \\
& +\sum_{k=2(i-1) \beta+1}^{2 i \beta}\left(\sum_{j=1}^{N}\left(a_{i, m} e^{j \phi_{i, m}} \gamma_{i}^{(j)} x_{k}^{(j)}\right) x_{k}^{(p)}\right) \\
& +\sum_{k=1}^{2 \beta} \xi_{k, m} x_{k}^{(p)} .
\end{aligned}
$$

Therefore MMSE will be

$$
\begin{aligned}
J_{\min } & =\operatorname{var}\left[\sum_{k=2(i-1) \beta+1}^{2 i \beta}\left(\sum_{j=1}^{N}\left(a_{i, m} e^{j \phi_{i, m}} \gamma_{i}^{(j)} x_{k}^{(j)}\right) x_{k}^{(p)}\right)\right. \\
& \left.+\sum_{k=1}^{2 \beta} \xi_{k, m} x_{k}^{(p)}\right] .
\end{aligned}
$$


Since both terms in the above equation are uncorrelated, therefore

$$
\begin{aligned}
J_{\min }= & a_{i, m}^{2} \sum_{k=2(i-1) \beta+1}^{2 i \beta}\left(\sum_{j=1}^{N} \operatorname{var}\left(x_{k}^{(j)} x_{k}^{(p)}\right)\right) \\
& +\sum_{k=1}^{2 \beta} \operatorname{var}\left(\xi_{k, m} x_{k}^{(p)}\right) \\
J_{\min }= & 2 \beta a_{i, m}^{2} N P_{c}^{2}+2 \beta N_{0} P_{c} .
\end{aligned}
$$

\section{B. Derivation of BER (See (17))}

Since the chaotic spreading sequences and AWGN noise are zero mean uncorrelated processes, hence

$$
\begin{aligned}
E & {\left[Z_{i}^{(g)} \mid \gamma_{i}^{(g)}=1\right] } \\
& =2 \beta E\left[\left(x_{k}^{(g)}\right)^{2}\right] \times \sum_{m=1}^{M} a_{i, m} \widehat{a}_{i, m} \cos \left(\phi_{i, m}-\widehat{\phi}_{i, m}\right) \\
& =2 \beta P_{c} \sum_{m=1}^{M} a_{i, m} \widehat{a}_{i, m} \cos \left(\phi_{i, m}-\widehat{\phi}_{i, m}\right) .
\end{aligned}
$$

Let (14) be represented as

$$
Z_{i}^{(g)}=Z_{i}^{a(g)}+Z_{i}^{b(g)}+Z_{i}^{c(g)},
$$

where

$$
\begin{aligned}
Z_{i}^{a(g)}= & \sum_{m=1}^{M} a_{i, m} \widehat{a}_{i, m} \cos \left(\phi_{i, m}-\widehat{\phi}_{i, m}\right) \\
& \times \sum_{k=2(i-1) \beta+1}^{2 i \beta} \gamma_{i}^{(g)}\left(x_{k}^{(g)}\right)^{2} \\
Z_{i}^{b(g)}= & \sum_{m=1}^{M} a_{i, m} \widehat{a}_{i, m} \cos \left(\phi_{i, m}-\widehat{\phi}_{i, m}\right) \\
& \times \sum_{k=2(i-1) \beta+1}^{2 i \beta} \sum_{j=1, j \neq g}^{N}\left(\gamma_{i}^{(j)} x_{k}^{(j)}\right) x_{k}^{(g)} \\
Z_{i}^{c(g)}= & \sum_{m=1}^{M} \widehat{a}_{i, m} \cos \left(\widehat{\phi}_{i, m}\right) \sum_{k=2(i-1) \beta+1}^{2 i \beta} \operatorname{Re}\left(\xi_{k, m}\right) x_{k}^{(g)} \\
& +\sum_{m=1}^{M} \widehat{a}_{i, m} \sin \left(\widehat{\phi}_{i, m}\right) \sum_{k=2(i-1) \beta+1}^{2 i \beta} \operatorname{Im}\left(\xi_{k, m}\right) x_{k}^{(g)} .
\end{aligned}
$$

All the three terms in (B.2) are uncorrelated to each other; hence $\operatorname{var}\left[Z_{i}^{(g)} \mid \gamma_{i}^{(g)}=1\right]$ is given by

$$
\begin{aligned}
\operatorname{var}\left[Z_{i}^{(g)} \mid \gamma_{i}^{(g)}=1\right]= & \operatorname{var}\left[Z_{i}^{a(g)}\right]+\operatorname{var}\left[Z_{i}^{b(g)}\right] \\
& +\operatorname{var}\left[Z_{i}^{c(g)}\right] .
\end{aligned}
$$

Variance of the first term $Z_{i}^{a(g)}$ can be written as

$$
\begin{gathered}
\operatorname{var}\left[Z_{i}^{a(g)}\right]=\operatorname{var}\left[\sum_{m=1}^{M} a_{i, m} \widehat{a}_{i, m} \cos \left(\phi_{i, m}-\widehat{\phi}_{i, m}\right)\right. \\
\left.\cdot \sum_{k=2(i-1) \beta+1}^{2 i \beta}\left(x_{k}^{(g)}\right)^{2}\right]=2 \beta\left\{\sum_{m=1}^{M} a_{i, m} \widehat{a}_{i, m}\right. \\
\left.\cdot \cos \left(\phi_{i, m}-\widehat{\phi}_{i, m}\right)\right\}^{2}\left[\operatorname{var}\left(x_{k}^{(g)}\right)^{2}\right] .
\end{gathered}
$$

Since all the chaotic sequences are mutually uncorrelated, hence variance of $Z_{i}^{b(g)}$ will be

$$
\operatorname{var}\left[Z_{i}^{b(g)}\right]=\operatorname{var}\left[\sum_{m=1}^{M} a_{i, m} \widehat{a}_{i, m} \cos \left(\phi_{i, m}-\widehat{\phi}_{i, m}\right)\right.
$$

$$
\begin{aligned}
& \left.\times \sum_{k=2(i-1) \beta+1}^{2 i \beta} \sum_{\substack{j=1, j \neq g \\
j=p}}^{N}\left(x_{k}^{(j)}\right) x_{k}^{(g)}\right] \\
& =2 \beta N\left\{\sum_{m=1}^{M} a_{i, m} \widehat{a}_{i, m} \cos \left(\phi_{i, m}-\widehat{\phi}_{i, m}\right)\right\}^{2} P_{c}^{2} .
\end{aligned}
$$

Real and Imaginary part of complex AWGN have power spectral density equal to $N_{0} / 2$ and independent from chaotic sequences; therefore variance of $Z_{i}^{c(g)}$ can be defined as

$$
\begin{aligned}
& Z_{i}^{c(g)} \\
& =\operatorname{var}\left[\sum_{m=1}^{M} \widehat{a}_{i, m} \cos \left(\widehat{\phi}_{i, m}\right) \sum_{k=2(i-1) \beta+1}^{2 i \beta} \operatorname{Re}\left(\xi_{k, m}\right) x_{k}^{(g)}\right. \\
& \left.+\sum_{m=1}^{M} \widehat{a}_{i, m} \sin \left(\widehat{\phi}_{i, m}\right) \sum_{k=2(i-1) \beta+1}^{2 i \beta} \operatorname{Im}\left(\xi_{k, m}\right) x_{k}^{(g)}\right] \\
& =\beta N_{0} P_{c} \sum_{m=1}^{M} \hat{a}_{i, m}^{2} .
\end{aligned}
$$

Putting (B.1), (B.5), (B.6), and (B.7) in (16) and after rearranging the equation we have (17). 


\section{BER Expression for Different Power and Multiplexed Pilot-Data}

If spreading sequence of pilot and data symbols has different power, then (17) can be rederived in the same manner as that for equal power case and final $P^{(g)}(a)$ equation will be

$$
\begin{gathered}
P^{(g)}(a)=\frac{1}{2} \operatorname{erfc}\left\{\left[\frac{\psi}{\beta}+\frac{(N-1)+P_{p} / P_{c}}{\beta}\right.\right. \\
+\left(\left(\frac{E_{b}}{N_{0}}\right)\right. \\
\left.\left.\left.. \frac{\left\{\sum_{m=1}^{M} a_{m} \widehat{a}_{m} \cos \left(\phi_{m}-\widehat{\phi}_{m}\right)\right\}^{2}}{\sum_{m=1}^{M} \hat{a}_{m}^{2}}\right)^{-1}\right]^{-1 / 2}\right\},
\end{gathered}
$$

where $P_{p}$ is the chip power of the pilot spreading sequence. It is clear from the above equation that as the pilot power increases the interuser interference increases, but at the same time there will be an improvement in the performance of the estimator. Simulation results show that increase in pilot power improves the overall performance of proposed CDMA system at low SNR condition. But the performance deteriorates at high SNR conditions.

Similarly for multiplexed pilot-data system, pilot is not creating any interuser interference and the final $P^{(g)}(a)$ can be defined as

$$
\begin{gathered}
P^{(g)}(a)=\frac{1}{2} \operatorname{erfc}\left\{\left[\frac{\psi}{\beta}+\frac{(N-1)}{\beta}+\left(\left(\frac{E_{b}}{N_{0}}\right)\right.\right.\right. \\
\left.\left.\cdot \frac{\left\{\sum_{m=1}^{M} a_{m} \widehat{a}_{m} \cos \left(\phi_{m}-\widehat{\phi}_{m}\right)\right\}^{2}}{\sum_{m=1}^{M} \widehat{a}_{m}^{2}}\right)^{-1}\right\} .
\end{gathered}
$$

Clearly the performance of this system will improve with increase in pilot power because the performance of estimator improves with high pilot power.

\section{Competing Interests}

The authors declare that they have no competing interests.

\section{References}

[1] V. Tarokh, H. Jafarkhani, and A. R. Calderbank, "Space-time block codes from orthogonal designs," IEEE Transactions on Information Theory, vol. 45, no. 5, pp. 1456-1467, 1999.

[2] S. M. Alamouti, "A simple transmit diversity technique for wireless communications," IEEE Journal on Selected Areas in Communications, vol. 16, no. 8, pp. 1451-1458, 1998.

[3] B. Sklar, "Rayleigh fading channels in mobile digital communication systems part II: mitigation," IEEE Communications Magazine, vol. 35, no. 9, pp. 148-155, 1997.
[4] B. Sklar, "Rayleigh fading channels in mobile digital communication systems part I: characterization," IEEE Communications Magazine, vol. 35, no. 7, pp. 90-100, 1997.

[5] L.-M. Chen and B.-S. Chen, "A robust adaptive DFE receiver for DS-CDMA systems under multipath fading channels," IEEE Transactions on Signal Processing, vol. 49, no. 7, pp. 1523-1532, 2001.

[6] W. G. Phoel and M. L. Honig, "Performance of coded DSCDMA with pilot-assisted channel estimation and linear interference suppression," IEEE Transactions on Communications, vol. 50, no. 5, pp. 822-832, 2002.

[7] P. Schramm, "Analysis and optimization of pilot-channelassisted BPSK for DS-CDMA systems," IEEE Transactions on Communications, vol. 46, no. 9, pp. 1122-1124, 1998.

[8] J.-S. Kim, H.-J. Shin, and D.-R. Shin, "Multiuser channel estimation using robust recursive filters for CDMA system," Journal of Communications and Networks, vol. 9, no. 3, pp. 219228, 2007.

[9] X. Wang and H. V. Poor, "Adaptive joint multiuser detection and channel estimation in multipath fading CDMA channels," Wireless Networks, vol. 4, no. 6, pp. 453-470, 1998.

[10] M.-A. R. Baissas and A. M. Sayeed, "Pilot-based estimation of time-varying multipath channels for coherent CDMA receivers," IEEE Transactions on Signal Processing, vol. 50, no. 8, pp. 2037-2049, 2002.

[11] G. Ganesan and P. Stoica, "Space-time diversity using orthogonal and amicable orthogonal designs," Wireless Personal Communications, vol. 18, no. 2, pp. 165-178, 2001.

[12] C. P. LaRosa, A. Mallette, J. P. Oliver, and B. W. Zancho, "Channel estimation in a RAKE receiver of a CDMA communication system," US Patent no. 7,428,262, September 2008, https://www.google.com/patents/US7428262.

[13] J. S. Nielsen, "Adaptive generalized matched filter RAKE receiver system and method," U.S. Patent No. 6,985,518.

[14] M. K. Patel, S. M. Berber, and K. W. Sowerby, "Bayesian channel estimation in chaos based multicarrier CDMA system under slowly varying frequency selective channel," International Journal of Circuits, Systems and Signal Processing, vol. 10, pp. 62-68, 2016.

[15] F. C. Lau and K. T. Chi, Chaos-Based Digital Communication Systems: Operating Principles, Analysis Methods, and Performance Evaluation, Springer, Berlin, Germany, 2003.

[16] G. S. Sandhu and S. M. Berber, "Investigation on orthogonal signals for secure transmission in multiuser communication," in Proceedings of the International Conference on Wireless Broadband and Ultra Wideband Communication (AusWireless '06), Sydney, Australia, 2006.

[17] R. Vali, S. M. Berber, and S. K. Nguang, "Effect of Rayleigh fading on non-coherent sequence synchronization for multiuser chaos based DS-CDMA," Signal Processing, vol. 90, no. 6, pp. 1924-1939, 2010.

[18] M. Coulon and D. Roviras, "Multi-user receivers for synchronous and asynchronous transmissions for chaos-based multiple-access systems," Signal Processing, vol. 89, no. 4, pp. 583-598, 2009.

[19] G. Kaddoum, A. J. Lawrance, P. Charge, and D. Roviras, "Chaos communication performance: theory and computation," Circuits, Systems, and Signal Processing, vol. 30, no. 1, pp. 185-208, 2011. 
[20] M. Coulon and D. Roviras, "Multi-user receivers for a multipleaccess system based on chaotic sequences on unknown asynchronous frequency-selective channels," Signal Processing, vol. 90, no. 2, pp. 587-598, 2010.

[21] G. Kaddoum, M. Coulon, D. Roviras, and P. Chargé, “Theoretical performance for asynchronous multi-user chaos-based communication systems on fading channels," Signal Processing, vol. 90, no. 11, pp. 2923-2933, 2010.

[22] G. Kaddoum, T. Lambard, and F. Gagnon, "Performance analysis of a chaos shift keying system with polarisation sensitivity under multipath channel," IET Communications, vol. 6, no. 12, pp. 1837-1845, 2012.

[23] G. Kaddoum, D. Roviras, P. Chargé, and D. Fournier-Prunaret, "Accurate bit error rate calculation for asynchronous chaosbased DS-CDMA over multipath channel," EURASIP Journal on Advances in Signal Processing, vol. 2009, Article ID 571307, 12 pages, 2009.

[24] M. Sangeetha and V. Bhaskar, "Performance analysis of subspace based downlink channel estimation for W-CDMA systems using chaotic codes," Wireless Personal Communications, vol. 71, no. 1, pp. 1-21, 2013.

[25] B.-Y. Wang and W. X. Zheng, "Adaptive blind channel equalisation in chaotic communications by using nonlinear prediction technique," IEE Proceedings-Vision, Image and Signal Processing, vol. 153, no. 6, pp. 810-814, 2006.

[26] Z. Zhu and H. Leung, "Adaptive blind equalization for chaotic communication systems using extended-Kalman filter," IEEE Transactions on Circuits and Systems I: Fundamental Theory and Applications, vol. 48, no. 8, pp. 979-989, 2001.

[27] C. Vural and G. Çetinel, "Blind equalization of single-input single-output fir channels for chaotic communication systems," Digital Signal Processing, vol. 20, no. 1, pp. 201-211, 2010.

[28] Z. Hu and J. Feng, "Blind channel equalization algorithm based on dual unscented Kalman filter for chaotic multi-input multioutput communication systems," Transactions of Tianjin University, vol. 18, no. 1, pp. 33-37, 2012.

[29] M. K. Patel, S. M. Berber, and K. W. Sowerby, "Bayesian channel estimation in chaos based ds-cdma system," in Proceedings of the International Conference on Electronics and Communication system, (ECS '15), pp. 60-64, Barcelona, Spain, April 2015.

[30] M. K. Patel, S. M. Berber, and K. W. Sowerby, "Adaptive RAKE receiver in chaos based pilot-added DS-CDMA system," Physical Communication, vol. 16, pp. 37-42, 2015.

[31] M. K. Patel, S. M. Berber, and K. W. Sowerby, "Performance analysis of adaptive chaos based CDMA system with antenna diversity in frequency selective channel," Wireless Personal Communications, vol. 84, no. 2, pp. 1439-1448, 2015.

[32] W. C. Jakes and D. C. Cox, Microwave Mobile Communications, Wiley-IEEE Press, Hoboken, NJ, USA, 1994.

[33] G. Kaddoum and F. Gagnon, "Performance analysis of STBCCSK communication system over slow fading channel," Signal Processing, vol. 93, no. 7, pp. 2055-2060, 2013.

[34] Z. Zvonar and M. Stojanovic, "Performance of antenna diversity multiuser receivers in CDMA channels with imperfect fading estimation," Wireless Personal Communications, vol. 3, no. 1-2, pp. 91-110, 1996.

[35] T. S. Rappaport, Wireless Communications: Principles and Practice, vol. 2, Prentice Hall PTR, Upper Saddle River, NJ, USA, 1996.

[36] S. Haykin, Adaptive Filter Theory, Prentice Hall, 4th edition, 2001.
[37] B. Jovic, C. P. Unsworth, G. S. Sandhu, and S. M. Berber, "A robust sequence synchronization unit for multi-user DSCDMA chaos-based communication systems," Signal Processing, vol. 87, no. 7, pp. 1692-1708, 2007.

[38] G. Kaddoum, D. Roviras, P. Chargé, and D. Fournier-Prunaret, "Robust synchronization for asynchronous multi-user chaosbased DS-CDMA," Signal Processing, vol. 89, no. 5, pp. 807-818, 2009.

[39] S. Haykin, Digital Communications, John Willey \& Sons, New York, NY, USA, 1988.

[40] J. S. Lee and L. E. Miller, CDMA Systems Engineering Handbook, Artech House, Boston, Mass, USA, 1998.

[41] C. C. Tan and N. C. Beaulieu, "On first-order Markov modeling for the rayleigh fading channel," IEEE Transactions on Communications, vol. 48, no. 12, pp. 2032-2040, 2000.

[42] H. S. Wang and P.-C. Chang, "On verifying the first-order Markovian assumption for a rayleigh fading channel model," IEEE Transactions on Vehicular Technology, vol. 45, no. 2, pp. 353-357, 1996.

[43] G. Kaddoum, P. Charg, D. Roviras, and D. Fournier-Prunaret, "Comparison of chaotic sequences in a chaos based DS-CDMA system," in Proceedings of the International Symposium on Nonlinear Theory and Its Applications, Vancouver, Canada, September 2007. 


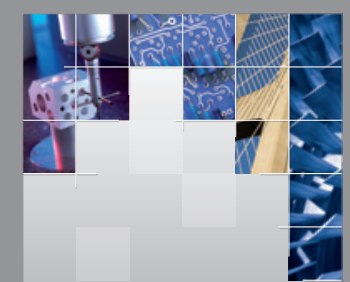

\section{Enfincering}
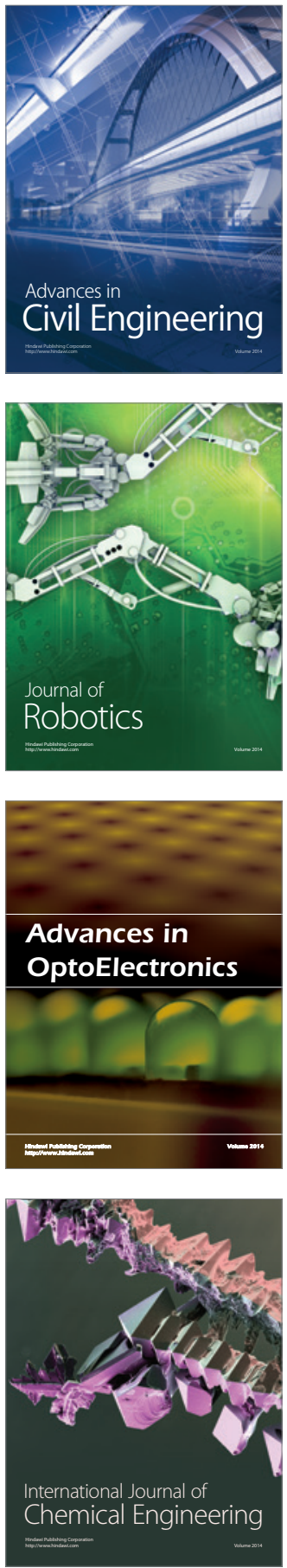

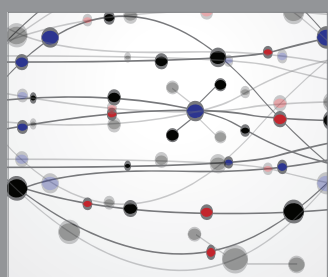

The Scientific World Journal

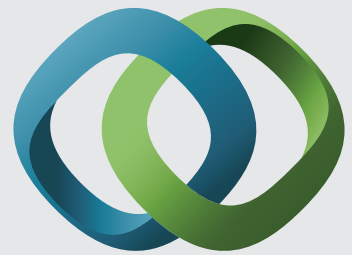

\section{Hindawi}

Submit your manuscripts at

https://www.hindawi.com
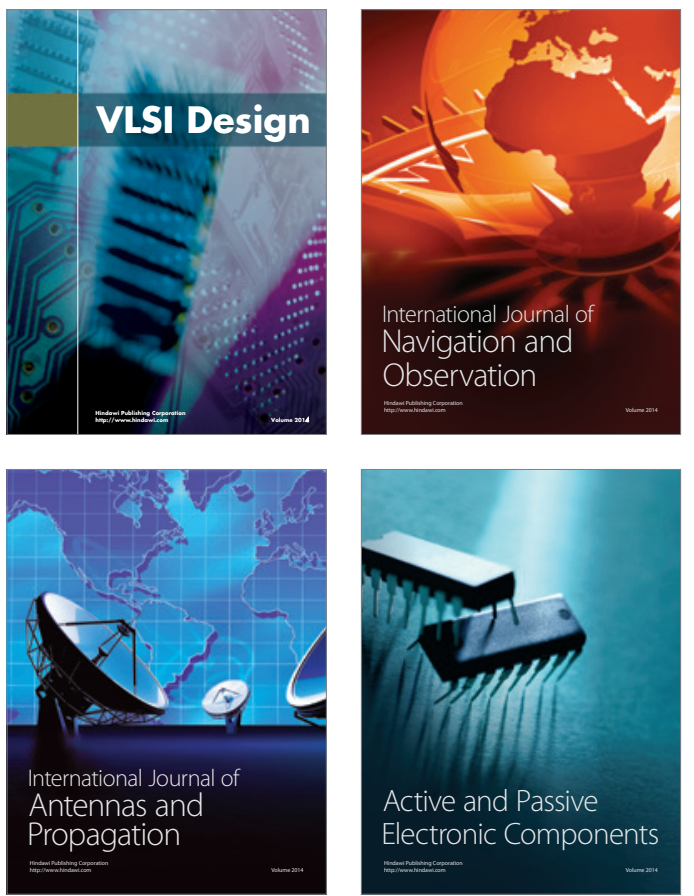
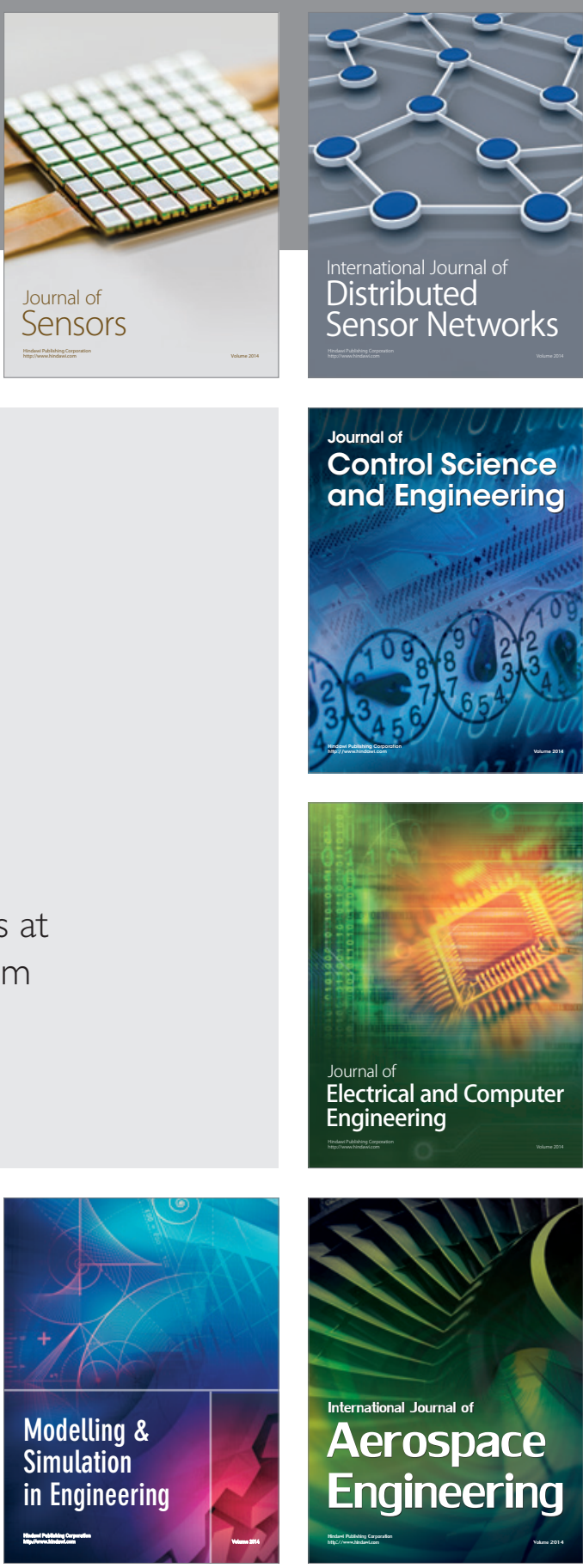

International Journal of

Distributed

Sensor Networks

$-$

Joumal of

Control Science

and Engineering
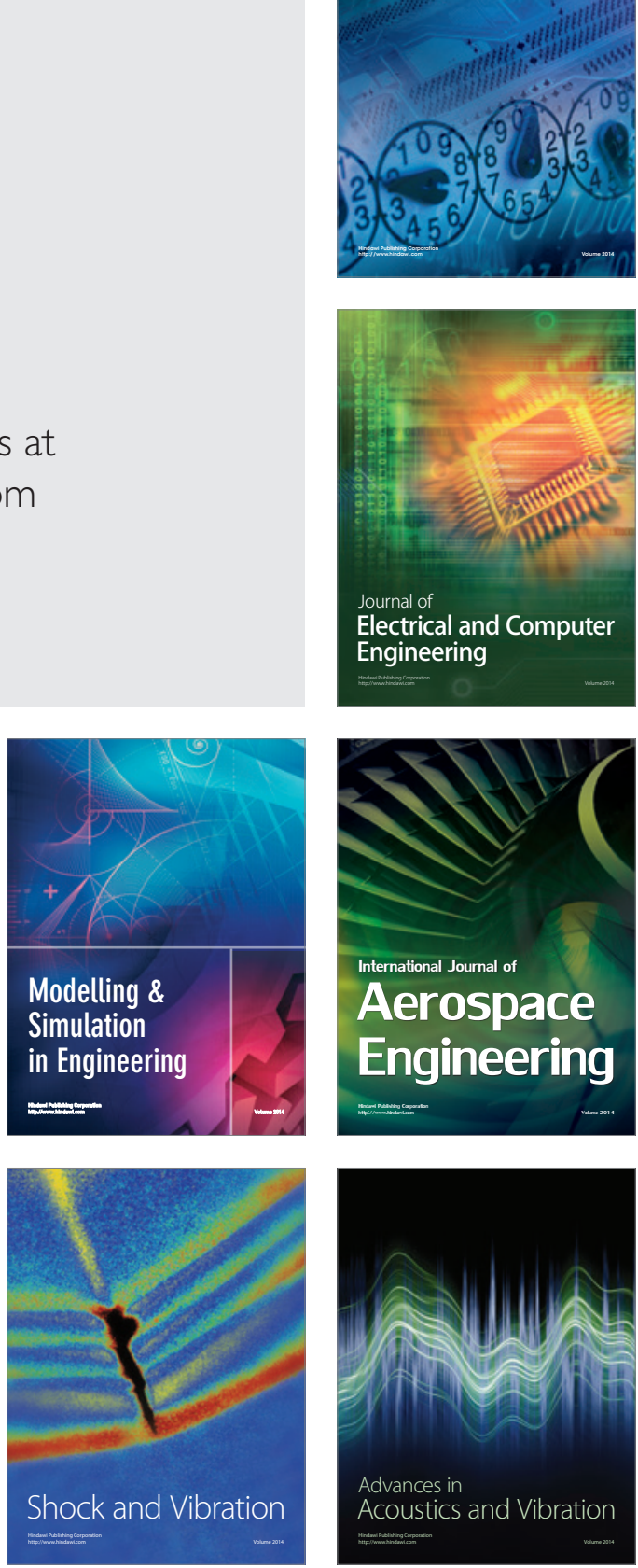